\title{
INTEGRATED PRODUCTION SCHEDULING AND DISTRIBUTION PLANNING WITH A TWO-STAGE SEMI- CONTINUOUS FLOW SHOP ENVIRONMENT
}

\author{
Yan, R. ; Li, M. M.,"\# \& Wei, W. C. ${ }^{* *}$ \\ *Donlinks School of Economic and Management, University of Science and Technology Beijing, \\ Beijing 100083, China \\ *School of Economic and Management, Beijing Jiaotong University, Beijing 100192, China \\ E-Mail: yr1900@163.com, limiaomiao@ustb.edu.cn,weiwenchao@bjtu.edu.cn \\ ( ${ }^{\#}$ Corresponding author)
}

\begin{abstract}
In the past decades, a significant amount of attention has been attracted by operational productiondistribution scheduling models. These models suggest that compared with hierarchical planning processes, the optimal plan can be achieved by considering the production and distribution decisions at the same time. For example, managers can schedule the production the delivery tasks consecutively. In a typical two-stage semi-continuous flow shop production plant, raw materials are converted into continuous resources in the first stage, and then, in the second stage, they are fed to the discreteproduction processes of end products. Machines are employed to guarantee the continuous running of certain types of processes in each stage. Furthermore, the processing sequence at the first stage determines the readiness time and costs of resources. The product family is the basic scheduling element, and products sharing common materials and manufacturing procedures are categorised in the same product family. Taking these dependencies derived in practical manufacturing processes into consideration, various industrial cases can be fit in this model, including the food and drink industries or the metallurgical industry. This paper finally presented numerical-based tests on specific cases from an industrial application.

(Received, processed and accepted by the Chinese Representative Office.)
\end{abstract}

Key Words: Operational Integrated Production-Distribution Scheduling, Two-Stage Production Process, Semi-Flexible Flow Shop, Sequence Dependent Setup Times and Costs

\section{INTRODUCTION}

In the industrial supply chain, raw materials are gradually converted into final products through a series of production stages, and then delivered to dispersed customers to meet their needs. With the globalization of the market, many enterprises have been trying to strictly optimize their production and distribution systems, thereby reducing the overall cost [1]. The extensive and sophisticated coordination among the various production processes in the supply chain is motivated by the incentives to pursue high overall performance of a system and to fulfil customers' requirements. Similarly, the efficiency and potentials saving can be significantly promoted by the integration among different supply chain functions $[2,3]$. Therefore, integrating different supply chain functions can significantly increase efficiency and save potential.

Over the last decades, the optimal operational-level integration has been researched by many studies. Nowadays, most papers mainly constraint their studies in a simple plant with a single machine at a single production level, such as an independent or parallel machine(s) [4]. Therefore, these papers fail to capture the dependency arguments that arise in the production scheduling sub-problem. For example, in a production plant with multiple production lines, the characteristics of the products being manufactured in a specified time period are determined by the type of raw materials being supplied to production system. The regular 
cleaning and maintenance of the production apparatus are also necessary to ensure the quality and purity of the products. The cost and duration for cleansing can usually be estimated according the sequence of product batches and the pre-set quality of the products. The production processes also often neglect the sequence-dependent setup operations [5]. It is clear that the dependencies described above significantly determine the detailed production planning at the operational level, and they can eventually affect the combined production and delivery scheduling at the end as well.

This study considers the manufacturing processes that are closely related to these dependencies. A two-stage production structure is suitable to model these processes. To be specific, the first stage is resource preparation at which raw materials are converted into continuous resources, and the second stage is product forming. The mass production of glass containers can be a great industrial example applying this model [6]. Each production line of these containers can reach a rate of thousands of products per minute. At the first stage, the mixture of raw materials, such as sand, soda ash, limestone and cullet is melted into approximately $1500^{\circ \mathrm{C}}$ glass paste, which is termed continuous resources in this field. The paste is then drawn into production lines at the second stage where they are shaped into final products - glass containers. Many other industrial applications can be fit in this production structure. Similarly, in the metallurgical industry [7, 8], it is common to transfer alloy into various vessels. The manufacturing of soft drinks can also be described by a two-stage production process [9], where the syrup and extracts are initially prepared in tanks, and at the second stage distributed to parallel bottling machines. Another example is the spinning industry. The fibres (of different fibre blends) are processed at the first production stage, which is then fed to parallel machines to manufacture different types of yarn [10]. In these examples, only the production-distribution planning procedure is able to apply the two-stage manufacturing structure, which may eventually change the production lot quantities, as well as the distribution timing and scaling [11]. The production sequences, lot sizes, vehicle routing plans should therefore be comprehensively balanced so that the system can achieve the optimised trade-offs between manufacturing and transportation costs, customer satisfaction, and tardiness penalties [12].

This paper investigates a model that integrates production and delivery scheduling at the operational level for a monthly planning horizon. The two-stage manufacturing structure can be regarded as a variation of the production-delivery models proposed by Park and Hong [13]. Considering the dependency constraints in the manufacturing stages, the synchronisation between the production and distribution scheduling is the primary modelling concern. Particularly, the costs and readiness times allocated at the initial production stage are considered to be dependent on the production sequence in this paper, while the costs and readiness times at the second stage only depend on the products. Section 3 verifies this modelling choice. The unsatisfied demands are either imposed on later production processes as a backlog, or penalized as lost sale if the supply-demand gap cannot be bridged within a certain time limit. In order to deal with the seasonally varied demand, companies usually produce a moderate amount of extra products in advance and store them in warehouses, but this can incur inventory costs. The distance between the manufacturer or warehouses and clients as well as the quantities of products being delivered determine the transportation costs. This model provides explicit values of backlog quantities, lost sale, inventory and delivery.

In this paper, operational decision-making problem in a plant with multiple production sites is tackled by the combination of production and distribution scheduling. According to certain types of production activities, production sites are selected correspondingly, and then they are matched with clients for delivering required quantities of products before the deadline. At present, no studies have thoroughly investigated an advanced production environment in this integrated context. The examination of the entire planning system should 
emphasise the detailed production planning needs. Specifically, this paper is interested in a semi-flexible two-stage production process where the outputs on each stage are related, and each customer order has to be processed by certain apparatus at each stage. Furthermore, various resource types at the initial stage and sequence-dependent costs and readiness times are taken into account in this paper.

\section{MODEL CONSTRUCTION}

\subsection{Assumptions}

(1) A plan of production and distribution is established every month, and production and distribution tasks are assigned to each day.

(2) Working hours are in days, and all products can be delivered after the second day of production.

(3) Switching product items is based on days. It is not available to switch product items and produce items on the same day. Switching product items is not allowed to be inter-period.

(4) The switching time between different families of products is an integer number of days, and the switching time between the same family products is zero.

(5) The production line that is undergoing production switching cannot be used to produce, and the production line can continue to work after the production switching is completed.

(6) Each customer specifies the time window for delivery of the goods (in days), and if the goods are delivered earlier or later than the specified time, a penalty fee is incurred.

(7) The client can be divided into multiple batches of delivery, and the same client can receive goods from different factories in batches.

(8) Customer needs can be met by different factories.

(9) Switching product items is not allowed at the beginning of each period, i.e. the product must be produced on the first day of the period.

The decision cycle here is day.

\subsection{Production scheduling model}

Main problem can be solved: the output at each period is known, and to get output of each day of this period.

To define indices, sets, parameters, and variables:

$D_{t}=\{1,2, \ldots, 20\}$, total number of working days in a period. $D_{i j m t} \subseteq D_{t}$, set of days to produce goods $i$ in plant $j$, with machine $m$ and at period $t . p_{m}$ : the number of product $i$ that can be processed on machine $m$ every day. $d$ : the $d^{\text {th }}$ working day at period $t, d \in D_{t} . x_{i i^{\prime} j m t}$ : $(=1)$ denotes that product $i$ is produced before product $i$, and between product $i$ and product $i$ ' there is no product to be produced (but there may be production switching, or production idle). In plant $j$, with machine $m$ and at period $t \cdot x_{i j m d t}:(=1)$ denotes that product $i$ is produced at $d^{\text {th }}$ day in plant $j$, with machine $m$, at period $t . x t_{j m d t}:(=1)$ denotes switching production at $d^{\text {th }}$ day in plant $j$, with machine $m$, at period $t . x w_{j m d t}:(=1)$ denotes that it is in a waiting state at $d^{\text {th }}$ day in plant $j$, with machine $m$, at period $t$.

Constraint conditions:

(1) $\sum_{i \in N} x_{i i^{\prime} j m t} \leq 1, \forall i^{\prime} \in N, \sum_{i^{\prime} \in N} x_{i i^{\prime} j m t} \leq 1, \forall i \in N$. There is only one production switching per kind of product per issue. $\sum_{i \in N} x_{i i^{\prime} j m t}=0$ denotes: don't produce goods $i$; $\sum_{i^{\prime} \in N} x_{i i^{\prime} j m t}=0$ denotes the last production produced in the same period.

(2) $\sum_{d \in D} x_{i j m d t}=x_{i j m t}$. The sum of production days of the current product $i$ equals total production days of current product $i$. In plant $j$, with machine $m$, at period $t$. 
(3) $\sum_{i \in N} x_{i j m d t} \leq 1$. Only one kind of product can be produced on the same day.

(4) $\sum_{i \in N} x_{i j m 1 t}=1$. Production switching or waiting is not allowed on the first day of each period, and only production is allowed.

(5) $\sum_{d \in D} \sum_{i \in N} x_{i j m d t}+\sum_{i^{\prime} \in N} \sum_{i \in N} x_{i i^{\prime} j m t} \cdot s t p_{i i^{\prime} j m t}+\sum_{d \in D} x w_{j m d t}=30$. The sum of production, switching and waiting days is equal to the total number of days in the current period.

(6) $\sum_{i^{\prime} \in N} \sum_{i \in N} x_{i i^{\prime} j m t}=\sum_{i \in N} \operatorname{sgn}\left(x_{i j m t}\right) \cdot\left|\sum_{i \in N} x_{i j m 30 t}-1\right|+\left(\sum_{i \in N} \operatorname{sgn}\left(x_{i j m t}\right)-1\right)$. $\sum_{i \in N} x_{i j m 30 t}$. The number of production switching at period $t$ is equal to the number of products categories in the current period, and the current period ends with production switching. That is, there is no product produced on the last day of the current period; the number of production switching in the period $t$ is equal to the number of production category in the current period minus one, and the product is produced on the last day of the current period. $S g n$ is a sign function (if $x_{i j m d t}>0$, it will return as 1 ; if $x_{i j m d t}=0$, it will return as 0 ; if $x_{i j m d t}<0$, it will return as -1 . $x_{i j m d t}$ can only be 1 or 0 , and cannot be less than 0 ).

(7) $\sum_{i \in f, i \in f^{\prime}, f \neq f^{\prime}} x_{i i^{\prime} j m t} \leq 1$. There can be only one production switching between different product families. That is, the products in the same family are switched into other family products after continuous production is finished.

(8) $x_{i j m d t}+x t_{j m d t}+x w_{\text {jmdt }}=1$. Only one of the situations of production, switching and waiting can occur on the same day.

(9) $\sum_{d \in D} x w_{j m d t}=s \cdot t_{s}-\sum_{d \in D} x t_{j m d t}$, if $s \cdot t_{s}>\sum_{d \in D} x t_{j m d t} ; \sum_{d \in D} x w_{j m d t}=0$, if $s \cdot t_{s} \leq \sum_{d \in D} x t_{j m d t}$. If the production switching time of feeding machine is more than it is on the manufacturing machine, there will be waiting state. The waiting time equals the difference of these two. Otherwise, the waiting time is 0 .

(10) $D_{i j m t}=\left\{d x_{i j m d t}=1, d \in D_{t}\right\}$.

(11) $\left(d^{\prime}-d\right) \cdot x_{i i^{\prime} j m t} \geq 0, \forall d \in D_{i j m t}, \forall d^{\prime} \in D_{i^{\prime} j m t}, i, i^{\prime} \in N$, switching from product $i$ to product $i$ ', the time of producing product $i$ is later than the time of producing product $i$ '.

(12) $\sum_{m \in M} \sum_{d \in D_{t}} x_{i j m d t} \cdot p_{i m}=\sum_{c \in C} S_{i j c t}$. The sum of the outputs is equal to the sum of the supplies.

\subsection{Logistics scheduling model}

Main problem can be solved: the quantities of supply and demand are known. Each plant has several vehicles, and a distribution plan is prepared for each vehicle. After the delivery task is completed on the same day, the vehicle can return to any plant.

$v \in V$ : vehicles. $G$ : maximum cargo load of the vehicle. $h_{i j}$ : inventory cost of plant $j$, product $i$ on each day. $V_{v j d t}:(=1)$, vehicle $v$ enters plant $j$, on the $d^{\text {th }}$ day, at the $t^{\text {th }}$ period. $V P_{v j d t}:(=1)$, vehicle $v$ enters plant $j$, on the $d^{\text {th }}$ day, at the $t^{\text {th }}$ period. The vehicle entering the factory on the same day will start distribution the next day. $V C_{v c d t}:(=1)$, vehicle $v$ enters client $c$, on the $d^{\text {th }}$ day, at the $t^{\text {th }}$ period to transport goods. $V N_{i v d t}:(=1)$, vehicle $v$ transport goods $i$, on the $d^{\text {th }}$ day, at the $t^{\text {th }}$ period; $(=0)$ means, the vehicle is idle on the same day. $V P_{v j d t} \cdot V N_{i v(d+1) t} \cdot V C_{v c(d+1) t}:(=1)$, denotes vehicle $v$ transport products $i$ from plant $j$ to client $c, \forall d \in D /\{20\}$.

Definition of vehicle transportation:

The client demand must be an integral multiple of the vehicle load. Each vehicle is loaded with only one kind of product at a time, and is fully filled.

(1) $\sum_{j \in P} V P_{v j d t}=1, \sum_{d \in D} \sum_{j \in P} V P_{v j d t}=20$. Each vehicle can only enter one factory on the same day and return to the factory every day. 
(2) $\sum_{c \in C} V C_{v c d t}=1$. Each vehicle can only deliver products to one client on the same day.

(3) $\sum_{i \in N} V N_{i v d t}=1$. Only one kind of product can be transported per vehicle per day.

(4) $V P_{v j 30 t}=V P_{v j 1(t+1)}$. The vehicle position of the $1^{\text {st }}$ day of period $t+1$ is same with the position of the last day of period $t$; the vehicle position of the $1^{\text {st }}$ period and $1^{\text {st }}$ day is predefined.

(5) $V N_{i v 1 t}=V C_{v c 1 t}=0$. All the vehicles are not assigned delivery tasks in the $1^{\text {st }}$ day of each period.

(6) $\sum_{d \in D} \sum_{v \in V} V N_{i v d t} \cdot V P_{v j d t} \cdot V C_{v c d t} \cdot G=S_{i j c t}$. Total amount of $i$ products which are delivered from plant $j$ to client $c$ equals current supply within the period $t$.

(7) $\sum_{\tau=1}^{d} \sum_{v \in V} V N_{i v \tau t} \cdot V P_{v j \tau t} \cdot G \leq \sum_{m \in M_{j}^{P}} \sum_{\tau=1}^{d} x_{i j m \tau t} \cdot p_{i m}+I_{i j(t-1)} \cdot$ Total transportation volume from the $1^{\text {st }}$ day to the $d^{\text {th }}$ day is no more than the sum of total output and inventory of the last period; we define product $i$, plant $j$ and period $t$; it is also assumed that the inventory of the $1^{\text {st }}$ period is 0 , as $I_{i j(t-1)}=0$.

The formulation of the cost of transportation:

$$
\begin{array}{r}
C T=\sum_{t \in T} \sum_{d \in D /\{30\}} \sum_{c \in C} \sum_{v \in V} \sum_{j \in P} \sum_{i \in N}\left(V_{v c(d+1) t}-V_{v j d t}+1\right) \cdot V N_{i v d t} \cdot r_{i j c} \\
+\sum_{t \in T} \sum_{d \in D} \sum_{c \in C} \sum_{v \in V} \sum_{j \in P} \sum_{i \in N}\left(V_{v c d t}-V_{v j d t}+1\right) \cdot V N_{i v d t} \cdot r_{i j c}
\end{array}
$$

The formula before plus sign means the distance from plants to clients. Formula after plus sign means the distance from clients to plants.

The formulation of the cost of inventory:

$$
\begin{aligned}
& C S=\sum_{t \in T} \sum_{d \in D} \sum_{m \in M} \sum_{j \in P} \sum_{i \in N}(20 T-d) \cdot x_{i j m d t} \cdot p_{i m} \cdot h_{i j} \\
&-\sum_{t \in T} \sum_{d \in D} \sum_{v \in V} \sum_{j \in P} \sum_{i \in N}(20 T-d) \cdot V N_{i v d t} \cdot p_{i m} \cdot h_{i j}
\end{aligned}
$$

The formula before minus sign means the inventory cost of products which aren't sold out since they were produced. Formula after minus sign means the cost weren't included in the calculated inventory cost after the goods are sold out.

\section{ALGORITHM DESIGN}

Production scheduling and vehicle scheduling are both classic NP-Hard problems. Heuristic algorithm is employed to determine the approximate optimal solution in large-scale applications. The joint optimization of production and delivery studied in this paper is also an NP-Hard problem. Since this problem cannot be directly solved by using the existing heuristic algorithms, a two-layer heuristic algorithm is proposed in this paper. Tabu search algorithm and particle swarm optimization are two well-known heuristic algorithms. There have been many research and applications on these two algorithms in recent years. The tabu search algorithm is faster, and has the advantages of high efficiency and strong effectiveness when dealing with the flow shop scheduling problems (FSSP), making the algorithm suitable as an outer optimization strategy. Although the particle swarm optimization algorithm has lower efficiency than the tabu search algorithm, it has the irreplaceable superiority due to its flexible coding methods and convenient variable neighbourhood search when solving some combinatorial optimization problems. Considering the facts discussed above, this paper developed an optimization strategy that integrates tabu search with particle swarm 
optimization. The tabu search algorithm is used to optimize the external production scheduling, while the particle swarm optimization algorithm is used to improve the internal vehicle scheduling.

\subsection{The strategy of tabu search algorithm}

The tabu algorithm (TA) is a meta-heuristic random search algorithm. The process initially starts from a feasible solution, and it selects a series of specific search directions or movements as trials. Then, it accepts a movement that can achieve the largest value change of the specific objective function. In order to avoid getting stuck in a local optimal solution, tabu search (TS) uses a flexible "memory" technology to record and select the optimization process and hence guided the next search direction. This is the establishment of the tabu table.

(1) Neighbourhood operation. In this paper, the neighbourhood of the tabu search algorithm is defined by three operations. Two of them are inter-plant operations, including relocation and exchange. The other one is an operation within the manufacturing plant called 2-Opt exchange. The relocation operation is able to ensure the feasibility of the solution by rescheduling a task to another manufacturing plant, and the rescheduled task would not exceed the capacity of the plant. The exchange operation is to select two tasks in two factories and exchange them with each other, and this process must also consider the feasibility of the solution. The 2-Opt exchange method is to delete the arcs that are not adjacent at both ends in the job sequencing, and then it replaces them with two new arcs.

(2) Tabu objects and tabu lengths. In this paper, the component variation of the tabu solution vector is employed as the tabu object. Each operation of a neighbourhood operation can be described by the collections of two sides: the inserted side and the deleted side. The two types of information are saved in a tabu table. If all sides involved in a specific operation are banned at the moment, then the operation is considered to be banned. The tabu lengths given to the inserted side and the deleted side are 5 and 9 respectively.

(3) Aspiration criteria. If an object in the tabu table satisfies the aspiration criteria, the tabu on that object is removed. The aspiration criteria applied in this paper are based on the evaluation values and the minimal error. The former one states that a solution can be excluded if its target value is better than any of the previous candidate optimal solutions. The later criterion states that the optimal solution in the current candidate solution set is excluded if all objects are considered as tabu objects.

\subsection{Particle swarm optimization}

Particle swarm optimization (PSO) was proposed by Kennedy and Eberhart [14] in 1995. The algorithm simulates the behaviour of birds in cluster flight, and enables the group to achieve the optimal goal through collective collaboration between birds [14]. The mathematical representation of the PSO algorithm is as follows: it is assumed that the search space is $D$ dimension, the number of total particles is $n$, and the position of the $I$ particle is expressed as a vector; the optimal position in the "flight" history of the $I$ particle (that is the optimal solution of the corresponding solution) is $P_{i}=\left(p_{i 1}, p_{i 2}, \ldots, p_{i D}\right)$. Among them, the optimal position of the $g$ particle in all of $P_{i}(i=1, \ldots, n)$, and the velocity of the $I$ particle is vector $V_{i}=\left(v_{i 1}, v_{i 2}, \ldots, v_{i D}\right)$.

The positions of each particle are changed according to the following formula:

$$
\begin{gathered}
v_{i d}(t+1)=w \cdot v_{i d}(t)+c_{1} \cdot \operatorname{rand}() \cdot\left(p_{i d}(t)-x_{i d}(t)\right)+c_{2} \cdot \operatorname{rand}() \cdot\left(p_{g d}(t)-x_{i d}(t)\right) \\
x_{i d}(t+1)=x_{i d}(t)+v_{i d}(t+1), 1 \leq i \leq n, 1 \leq d \leq D
\end{gathered}
$$

Among them, $c_{1}, c_{2}>0$ is the acceleration factor; $\operatorname{rand}()$ is the random number between $[0,1] ; \operatorname{rand}()$ is the inertia factor, the larger range exploration is suitable for the solution space, 
and the smaller one is suitable for the small range exploitation. The range of position change of the $d(1 \leq d \leq D)$ dimension is $\left[-X_{\max }, X_{\max }\right]$, the range of velocity change is $\left[-\operatorname{Vmax}_{d}, \operatorname{Vmax}_{d}\right]$. In the iteration process, the position and velocity exceed the boundary value.

\section{SIMULATION EXPERIMENT}

At present, there is no standard test case that can be directly applied, so this paper randomly gives a demonstration example to demonstrate the optimization process and analyse the optimization results. Suppose there are two factories, Factory 1 has one machine; factory 2 has two machines, two products in total. The capacity of each machine is shown in Table I.

Table I: Productivity parameter of simulation example.

\begin{tabular}{|c|c|c|c|}
\hline Plant & Machine & Production & Productivity \\
\hline 1 & 1 & 1 & 2 \\
\hline 1 & 1 & 2 & 1 \\
\hline 2 & 1 & 1 & 2 \\
\hline 2 & 1 & 2 & 2 \\
\hline 2 & 2 & 1 & 3 \\
\hline 2 & 2 & 2 & 2 \\
\hline
\end{tabular}

Table II: Location of plant and customer and demand of production.

\begin{tabular}{|clc|ccc|}
\hline No. & Location & Demand (1, 2) & No. & Location & Demand (1, 2) \\
\hline 1 & $(8.5,12)$ & $(5,5)$ & 7 & $(11,9)$ & $(7,4)$ \\
\hline 2 & $(17.5,15)$ & $(5,8)$ & 8 & $(18.5,10)$ & $(3,5)$ \\
\hline 3 & $(10.5,14.5)$ & $(4,8)$ & 9 & $(16,13)$ & $(2,3)$ \\
\hline 4 & $(12.5,10)$ & $(4,6)$ & 10 & $(15,7.5)$ & $(5,4)$ \\
\hline 5 & $(6.5,1.5)$ & $(2,10)$ & Plant 1 & $(8,8)$ & - \\
\hline 6 & $(7.5,13.5)$ & $(3,7)$ & Plant 2 & $(15,15)$ & - \\
\hline
\end{tabular}

They have 5 cars with the same capacity of 20 can be invoked, and the driving cost per unit distance is 1 . There are 10 customers; factory and customer coordinates are shown in Table II. The decision cycle is 3, and each cycle has 20 working days. Each customer has the same demand in different periods. Let $G=10, h_{i j}=2, r_{i j c}=1$.

The above data are substituted into the model and solved by the two stage optimization algorithm. The algorithm parameters are set as: $c_{1}=2, c_{2}=2, w=1.5$, and the maximum number of iterations is 1000 . Matlab R2017b was used for programming. The operating environment was: Core i7, 4.9 Ghz, 32 GB RAM and Windows 10. In order to better reflect the performance of the algorithm, the algorithm runs 10 times with an average running time of 10.8 seconds. The convergence process of the optimal results is shown in Fig. 1. It can be seen that the algorithm quickly converges to the optimal solution, then after more than 400 generations to jump out of the local optimal, to find a better solution, which shows that the algorithm has good convergence and search ability.

The final production and distribution plan is shown in Fig. 2. It can be seen from Fig. 2 that whole needs of all customers have been satisfied. The same product is often manufactured continuously across cycles to reduce production conversion costs. The production cost of the third cycle is higher than the previous two cycles, but the production conversion cost is lower, so the total cost is lower than the previous two cycles. 


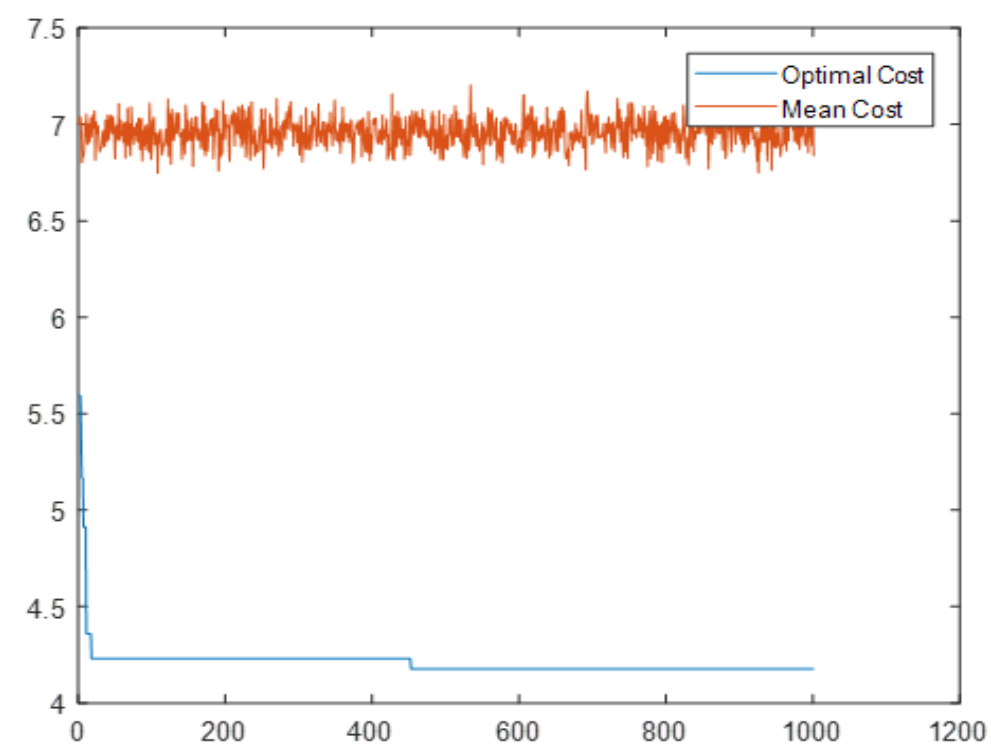

Figure 1: Convergence process graph of algorithm.
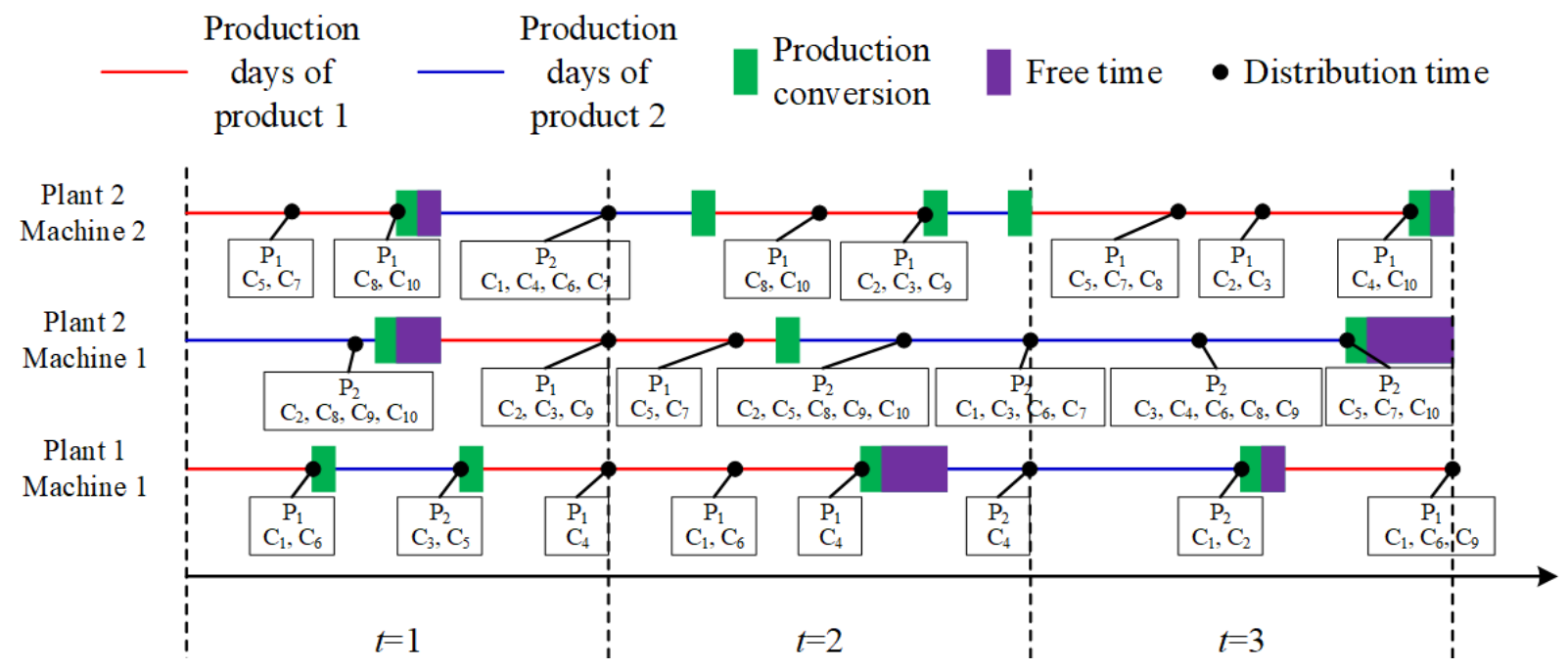

Figure 2: An illustrative production and distribution planning.

\section{CONCLUSIONS}

We consider a two-stage semi-continuous flow shop production environment. In the first step, the raw materials are converted into the resources required in the second stage through the corresponding procedures, and help to disperse the final products and machines in each stage are dedicated to certain types of processes. In addition, the order in which the raw materials are processed in the first step will determine the setup cost and time. The minimum scheduling unit is determined by the product family and shared resources. We constructed the production scheduling model and the logistics distribution model separately, and linked the two models through inventory parameters. A two stage heuristic algorithm is based on tabu search algorithm and particle swarm optimization algorithm. The tabu search algorithm is used to optimize the outer production scheduling problem, and particle swarm optimization is suitable for solving the problem of optimizing vehicle inner-layer scheduling. The simulation results show that the optimization algorithm is able to get the approximate optimal solution of the optimization problem. The models and algorithms in this paper are suitable for industrial production cases. More effective measures will also be developed to cut down solution space size and speed up problem solving approaches. 


\section{ACKNOWLEDGEMENT}

The work has been funded by National Natural Science Foundation of China (71802021, 71602008, 7161101015), the Beijing Municipal Natural Science Foundation (9184023), Beijing Social Science Fund Research Project (16JDGLC032, 17JDGLB011, 18GLB022), Fundamental Funds for Humanities and Social Sciences of Beijing Jiaotong University (2017jbwy004), and Fundamental Research Funds for the Central Universities (FRF-OT-18-012).

\section{REFERENCES}

[1] Thomas, D. J.; Griffin, P. M. (1996). Coordinated supply chain management, European Journal of Operational Research, Vol. 94, No. 1, 1-15, doi:10.1016/0377-2217(96)00098-7

[2] Geismar, H. N.; Laporte, G.; Lei, L.; Sriskandarajah, C. (2008). The integrated production and transportation scheduling problem for a product with a short lifespan, INFORMS Journal on Computing, Vol. 20, No. 1, 21-33, doi:10.1287/ijoc.1060.0208

[3] Reimann, M.; Tavares Neto, R.; Bogendorfer, E. (2014). Joint optimization of production planning and vehicle routing problems: A review of existing strategies, Pesquisa Operacional, Vol. 34, No. 2, 189-214, doi:10.1590/0101-7438.2014.034.02.0189

[4] Moons, S.; Ramaekers, K.; Caris, A.; Arda, Y. (2017). Integrating production scheduling and vehicle routing decisions at the operational decision level: A review and discussion, Computers \& Industrial Engineering, Vol. 104, 224-245, doi:10.1016/j.cie.2016.12.010

[5] Chen, Z.-L.; Vairaktarakis, G. L. (2005). Integrated scheduling of production and distribution operations, Management Science, Vol. 51, No.4, 614-628, doi:10.1287/mnsc.1040.0325

[6] Almada-Lobo, B.; Oliveira, J. F.; Carravilla, M. A. (2008). Production planning and scheduling in the glass container industry: A VNS approach, International Journal of Production Economics, Vol. 114, No. 1, 363-375, doi:10.1016/j.ijpe.2007.02.052

[7] De Araujo, S. A.; Arenales, M. N.; Clark, A. R. (2007). Joint rolling-horizon scheduling of materials processing and lot-sizing with sequence-dependent setups, Journal of Heuristics, Vol. 13, No. 4, 337-358, doi:10.1007/s10732-007-9011-9

[8] Dos Santos-Meza, E.; dos Santos, M. O.; Arenales, M. N. (2002). A lot-sizing problem in an automated foundry, European Journal of Operational Research, Vol. 139, No. 3, 490-500, doi:10.1016/S0377-2217(01)00196-5

[9] Ferreira, D.; Morabito, R.; Rangel, S. (2009). Solution approaches for the soft drink integrated production lot sizing and scheduling problem, European Journal of Operational Research, Vol. 196, No. 2, 697-706, doi:10.1016/j.ejor.2008.03.035

[10] Camargo, V. C. B.; Toledo, F. M. B.; Almada-Lobo, B. (2014). HOPS - Hamming-Oriented Partition Search for production planning in the spinning industry, European Journal of Operational Research, Vol. 234, No. 1, 266-277, doi:10.1016/j.ejor.2013.10.017

[11] Toraman, Ç.; Karadağ, E. (2018). Analyzing process of problem solving with group via statistical discourse analysis, Educational Sciences: Theory \& Practice, Vol. 18, No. 2, 229-252, doi:10.12738/estp.2018.2.0309

[12] Song, S.-L. (2018). Application of gray prediction and linear programming model in economic management, Mathematical Modelling of Engineering Problems, Vol. 5, No. 1, 46-50, doi:10.18280/mmep.050107

[13] Park, Y.-B.; Hong, S.-C. (2009). Integrated production and distribution planning for single-period inventory products, International Journal of Computer Integrated Manufacturing, Vol. 22, No. 5, 443-457, doi:10.1080/09511920802527590

[14] Kennedy, J.; Eberhart, R. (1995). Particle swarm optimization, Proceedings of the ICNN'95 International Conference on Neural Networks, Vol. 4, 1942-1948, doi:10.1109/ $\underline{\text { ICNN.1995.488968 }}$ 